\title{
Continuum mathematics at the nanoscale
}

\author{
Tim G Myers ${ }^{1 *}$, Michelle M MacDevette ${ }^{1,2}$, Francesc Font ${ }^{1,2}$ and Vincent Cregan ${ }^{1}$
}

\author{
*Correspondence: tmyers@crm.cat \\ ${ }^{1}$ Centre de Recerca Matemàtica, \\ Campus de Bellaterra, Edifici $C$, \\ Bellaterra, Barcelona, 08193, Spain \\ Full list of author information is \\ available at the end of the article
}

\begin{abstract}
In this paper we discuss three examples where continuum theory may be applied to describe nanoscale phenomena:

1. Enhanced flow in carbon nanotubes (CNTs) - This model shows that the experimentally observed enhancement can be explained using standard flow equations but with a depletion layer between the liquid and solid interfaces.

2. Nanoparticle melting - Nanoparticles often exhibit a sharp increase in melting rate as the size decreases. A mathematical model will be presented which predicts this phenomena.

3. Nanofluids - Experimental results concerning the remarkable heat transfer characteristics of nanofluids are at times contradictory. We develop a model for the thermal conductivity of a nanofluid, which provides much higher predictions than the standard Maxwell model and a better match to data.
\end{abstract}

Keywords: carbon nanotubes; enhanced flow; nanoparticle melting; thermal conductivity; nanofluid

\section{Introduction}

Continuum theory may be applied when there is a sufficiently large sample size to ensure that statistical variation of material quantities, such as density, is small. For fluids the variation is often quoted as $1 \%$ [1]. Assuming a spherical sample Nguyen and Werely [2] suggest this level of variation requires a minimum of $10^{4}$ atoms and so deduce a critical dimension of the order 10 and $90 \mathrm{~nm}$ for liquids and gases respectively. In fact by comparing molecular dynamics (MD) simulations to computations based on the Navier-Stokes equations Travis et al. [3] show that continuum theory may be applied to water flow down to around $3 \mathrm{~nm}$. Thomas et al. [4] suggest a figure of $1.66 \mathrm{~nm}$. In the field of heat transfer and phase change it has been suggested that continuum theory requires particle radii greater than $2 \mathrm{~nm}$ [5]. Kofman et al. [6] state that at scales smaller than $5 \mathrm{~nm}$ the melting process is discontinuous and dominated by fluctuations, Kuo and Clancy [7] observed structural changes and a 'quasi-molten' state in their study of nanoparticle melting between 2-5 nm.

Nanoscale is typically described as involving materials with at least one dimension below $100 \mathrm{~nm}$ [8], so there is clearly a range of sizes where continuum theory may be applied to nano phenomena. In this paper we will discuss work carried out in the Industrial Mathematics Group at the Centre de Recerca Matemàtica using continuum theory to study problems in fluid and heat flow and demonstrate how seemingly anomalous behaviour may be explained without resorting to molecular dynamics or empirically based adjustments. Full details of the group's work in this area may be found in [9-14]. In Section 2

\section{Springer}

○2014 Myers et al.; licensee Springer. This is an Open Access article distributed under the terms of the Creative Commons Attribution License (http://creativecommons.org/licenses/by/2.0), which permits unrestricted use, distribution, and reproduction in any medium, provided the original work is properly cited. 
we will examine the issue of enhanced flow in carbon nanotubes. Many experiments have shown that when water flows through a carbon nanotube the flow rate is orders of magnitude higher than predicted by classical theory. Early papers $[15,16]$ quote factors of around 4 orders of magnitude, although more recent experiments have shown the true figure is closer to 1 order [17]. In our model we employ a concept taken from non-Newtonian fluid dynamics, that of a bi-viscosity fluid, to explain why observed flow rates of water in carbon nanotubes are much higher than that predicted by classical theory. The model also suggests a physical interpretation for the Navier slip condition. In Section 3 we investigate the melting of nanoparticles. As the particles decrease in size, and so the ratio of bulk to surface atoms decreases, it becomes easier for surface atoms to leave the particle. This results in a decrease in the melt temperature. Using a form of the Gibbs-Thompson relation to describe the variation of melt temperature with size we produce a model that explains the experimentally observed 'abrupt melting. The final problem concerns nanofluids, these are fluids containing a suspension of nanoparticles. They have been claimed to significantly increase certain fluid properties, such as the thermal conductivity and heat transfer coefficient. Despite hundreds of papers affirming the remarkable heat transfer properties, it was recently shown in a benchmark study, carried out in over thirty laboratories around the world, that nanofluids do not provide significant improvements [18]. A theoretical demonstration that increasing nanoparticle concentration can decrease heat transfer was shown in [19]. This contradicted many analytical papers using the same model. However, experiments seem clear that the conductivity of the fluids increases significantly with particle concentration. The classical Maxwell model, to describe the heat conduction of a solid-in-liquid suspension, is known to significantly underpredict the thermal response of a nanofluid. In Section 4 we investigate the thermal conductivity of a nanofluid. The Maxwell model is based on a static analysis. Using an approximate solution method to the heat flow problem we obtain an expression for the thermal conductivity of the fluid which shows much better agreement with experiment.

A review on the mathematics of the continuum to describe behaviour at the nanoscale can be found in [20]. Previous mathematical models describing the melting of nanoparticles based on continuum theory exist, for instance [21-23].

\section{Enhanced flow in carbon nanotubes}

Carbon nanotubes (CNTs) are remarkably strong and light cylindrical nanostructures which are excellent at conducting both heat and electricity. These advantageous properties have led to CNTs being proposed for a diverse range of applications (see [24]) including DNA gene delivery [25], biosensor diagnostics [26], tissue engineering [27], solar cells [28] and microelectronics technology (e.g., transistors [29]). CNTs have also shown promise in the textile field, as they can transport water much quicker than that predicted by classical flow theory. Several authors have shown that by using CNTs, flow can be increased by up to several orders of magnitude $[15,16]$. However, more recently Whitby et al. [17] reported a more conservative maximum increase by a factor of 45 . Following the work by Myers [11], here we demonstrate that the inclusion of a gas depletion layer in the standard flow model accounts for the aforementioned enhanced increase in flow. 
Fluid flow in a circular pipe is explained by the well-known Hagen-Poiseuille equation which yields the fluid flux expression

$$
Q_{\mathrm{HP}}=-\frac{\pi R^{4} p_{z}}{8 \mu},
$$

where $R$ is the pipe radius, $p_{z}$ is the pressure gradient along the pipe and $\mu$ the fluid viscosity. Research has demonstrated that the flux associated with CNTs is considerably higher than (1). To explain this enhancement, a typical approach is to replace the no-slip boundary condition, $u(R)=0$, with the slip length formulation

$$
u(R)=-L_{s} \frac{\partial u(R)}{\partial r}
$$

where $L_{s}$ is the slip length. This leads to a modified flux expression

$$
Q_{\text {slip }}=Q_{\text {HP }}\left(1+\frac{4 L_{s}}{R}\right) \text {. }
$$

Flow enhancement is generally defined as, $\varepsilon_{\text {slip }}=Q_{\text {slip }} / Q_{\mathrm{HP}}$, the ratio of the observed to predicted fluxes, and thus $L_{s}$ has an obvious effect on any enhancement in the flow. On the microscale, it has been shown experimentally, that the slip length is much smaller than the channel dimension. In contrast, the slip length for nanochannels is usually of the order of microns. At present, there is no accepted theory for the slip length of a liquid flowing past a solid surface. However, there is one for gases, and the slip length is of the order of the mean free path of the gas [30]. Holt et al. [15] and Majumder et al. [16] report slip lengths of the order of microns to match with their corresponding experimental treatments. However, some authors [31,32] have questioned the validity of the slip modified Hagen-Poiseuille model based on the larger slip lengths reported in CNT studies. Cottin-Bizonne et al. [33] suggest that the slip length should have a single, radius-independent value and be much smaller than those typically quoted in the literature. In addition, they postulate that the contamination by hydrophobic particles to be the cause of some of the high experimental values. The hydrophobicity of CNTs has been proposed as an alternative explanation to the slip length. Eijkel and van den Berg [34] report that the strength of attraction between water molecules is greater than the attractive force between the hydrophobic solid and the water. This hydrophobicity can then lead to some form of 'depletion layer' which may be interpreted as a region of low viscosity close to the tube wall. Experimentally this may be viewed in terms of 'apparent' slip [34,35]. Several authors have observed this phenomenon experimentally [36-38] and via molecular dynamics simulations [39].

We can model the scenario where a fluid flows over a depletion layer by assuming a biviscosity flow model. Specifically, the model consists of equations for the bulk flow region in the centre of the channel and a depleted region with low viscosity at the walls of the tube. Matching the velocity and shear stress at the interface of these two regions at $r=\alpha$ results in the flux expression

$$
Q_{\mu}=Q_{\mathrm{HP}} \frac{\alpha^{4}}{R^{4}}\left(1+\frac{\mu_{1}}{\mu_{2}}\left(\frac{R^{4}}{\alpha^{4}}-1\right)\right),
$$

where $\mu_{1}, \mu_{2}$ denote the bulk and depletion layer viscosities, respectively, such that $\mu_{1} \gg \mu_{2}$. Experimental studies on CNTs indicate a depletion later thickness of $\delta=0.7 \mathrm{~nm}$, 
and thus $\alpha=R-0.7 \mathrm{~nm}$. The flow enhancement is defined as the ratio $\varepsilon_{\mu}=Q_{\mu} / Q_{\mathrm{HP}}$. If we take experimental data from Whitby et al. [17] we obtain $\mu_{2} \approx 0.018 \mu_{1}$. Two readily available gases, air and oxygen, have viscosities approximately 0.02 that of water.

As highlighted, gas flow theory accounts for apparent slip over a solid surface, but no equivalent theory exists for fluids. By comparing the flux expressions using a slip model and a depletion layer we obtain an expression for the slip length

$$
L_{s}=\delta\left(\frac{\mu_{1}}{\mu_{2}}-1\right)\left[1-\frac{3}{2} \frac{\delta}{R}+\left(\frac{\delta}{R}\right)^{2}-\frac{1}{4}\left(\frac{\delta}{R}\right)^{3}\right]
$$

see [11]. As predicted by Thomas and McGaughey [31] the slip length is a monotonically decreasing function of $R$. In addition, noting that $\mu_{1} / \mu_{2} \gg 1$, we can identify three distinct tube regimes, namely: wide, moderate and small tubes.

1. For sufficiently wide tubes where $\delta / R \ll \mu_{2} / \mu_{1}$ we have $\varepsilon_{\mu} \approx 1$, and there is no noticeable flow enhancement. As a result the no-slip boundary condition is sufficient. Slip is not observed in wide tubes with smooth surfaces. This condition holds for $R>3 \mu \mathrm{m}$.

2. For moderate tubes where $(\delta / R)\left(\mu_{1} / \mu_{2}\right)$ is $\mathcal{O}(1)$ but $\delta / R \ll 1$ then only the leading order term of $L_{s}$ applies and

$$
\varepsilon_{\mu} \approx 1+\frac{4 \delta}{R}\left(\frac{\mu_{1}}{\mu_{2}}-1\right)
$$

This applies approximately for $R \in[21 \mathrm{~nm}, 3 \mu \mathrm{m}]$, and reflects a constant slip length of $L_{s}=\delta \mu_{1} / \mu_{2}$. Several authors $[33,40]$ describe constant slip-lengths around $20-40 \mathrm{~nm}$.

3. For very small tubes where $\delta / R$ is $\mathcal{O}(1)$ we require the full expression for $\varepsilon_{\mu}$, and thus the slip length varies with $R$.

The $R$ bounds for the different regimes are chosen such that there is a maximum error of $5 \%$ when using the approximation.

The present model predicts $\varepsilon_{\mu} \approx 33.2$ for $R=3.5 \mathrm{~nm}$ which is corroborated by Thomas et al. [4] who report $\varepsilon_{\mu} \approx 32$. The model also predicts a maximum enhancement (obtained by setting $R=\delta$ ) of approximately 50 which agrees with the maximum value of 45 recorded by Whitby et al. [17].

\section{Nanoparticle melting}

Nanoparticles have a large ratio of surface to volume atoms which can have a significant impact on the material properties [5]. One example where this manifests itself is in the well-known decrease in phase change temperature that accompanies a decrease in material dimensions [41]. The experimental treatment of Buffat and Borel [42] documented a decrease of approximately $500 \mathrm{~K}$ for gold particles with radius slightly greater than $1 \mathrm{~nm}$. Similarly, the MD simulations of Shim et al. [41] demonstrated a decrease of more than $800 \mathrm{~K}$ below the bulk melt temperature for gold nanoparticles with a radius around $0.8 \mathrm{~nm}$. This interesting property has resulted in nanoparticles being the subject of research for applications which require the particles to melt after serving their primary purpose, and so pass through the system as disperse molecules. Drugs with poor water solubility may be administered as nanoparticles to improve their uptake. Bergese et al. [43] and Liu et 
al. [44] consider antibiotic and antianginal drugs, which exhibit a melting point depression of around $30 \mathrm{~K}$. Since gold has low toxicity, gold nanoparticles also make good carriers for drug and gene delivery [45]. Hence, an accurate model to understand the thermal behaviour of a nanoparticle and its probable phase change behaviour is extremely desirable. In this section we present a mathematical model describing the melting process of nanoparticles proposed by Font and Myers [12].

Assuming that density and specific heat are approximately constant in both the solid and liquid phases, the melt temperature is obtained via the generalised Gibbs-Thomson relation

$$
L_{m}\left(\frac{T_{m}}{T_{m}^{*}}-1\right)+\Delta c\left[T_{m} \ln \left(\frac{T_{m}}{T_{m}^{*}}\right)+T_{m}^{*}-T_{m}\right]=-\frac{2 \sigma_{s l} \kappa}{\rho}
$$

where $L_{m}$ is the latent heat, $T_{m}$ is the temperature at which the phase change occurs, $T_{m}^{*}$ the bulk phase change temperature, $\Delta c=c_{l}-c_{s}$ the change in specific heat from liquid to solid, $\sigma_{s l}$ the surface tension and $\kappa$ the mean curvature. In the present model we assume equal density for the solid and liquid phases, which is denoted by $\rho$. For the present analysis we assume that the ambient pressure variation is small, and thus a pressure related term which may appear in this relation is assumed negligible. Figure 1 compares the generalised Gibbs-Thomson relation against experimental data for gold nanoparticles in the range 2$12 \mathrm{~nm}$, see [12].

For the melting of a spherically symmetric particle we consider the dimensionless model

$$
\frac{\partial T}{\partial t}=\frac{1}{r^{2}} \frac{\partial}{\partial r}\left(r^{2} \frac{\partial T}{\partial r}\right), \quad \frac{\partial \theta}{\partial t}=\frac{k}{c} \frac{1}{r^{2}} \frac{\partial}{\partial r}\left(r^{2} \frac{\partial \theta}{\partial r}\right)
$$

where $T$ and $\theta$ denote the temperature in the liquid and solid, and $k, c$ are the solid to liquid conductivity and specific heat ratios, respectively. The boundary conditions are $T(1, t)=1$, $T(R(t), t)=\theta(R(t), t)=T_{m},\left.\frac{\partial \theta}{\partial r}\right|_{r=0}=0$, where $r=R(t)$ is the position of the solid-liquid interface. The Stefan condition is

$$
\left[\beta+(1-c) T_{m}\right] \frac{d R}{d t}=\left.k \frac{\partial \theta}{\partial r}\right|_{r=R}-\left.\frac{\partial T}{\partial r}\right|_{r=R}
$$

Figure 1 Melt temperature versus radius of gold nanoparticles. Circles show experimental data, the solid line is the generalised Gibbs-Thomson relation and the dashed line the Gibbs-Thomson relation with $\Delta c=0$. Figure from [12].

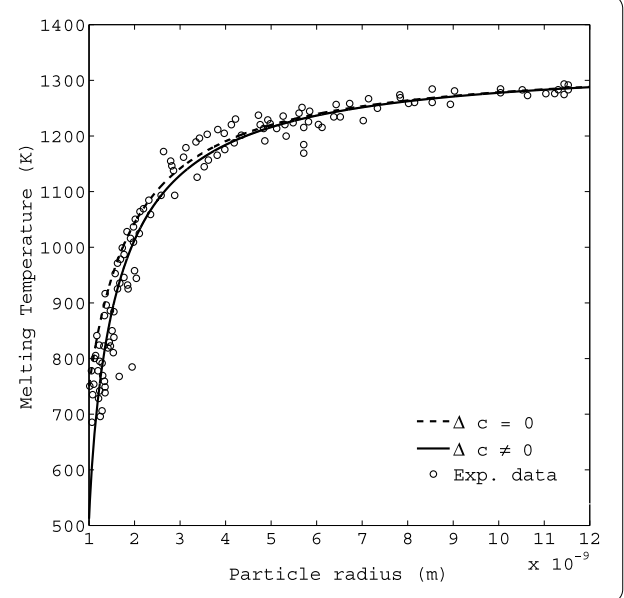


This model is similar to that described in [23]: the main difference being that our GibbsThomson relation includes $\Delta c$. As will be seen later, for large particles this does not greatly affect the results but becomes increasingly important as the particle size decreases.

The dimensionless melting temperature $T_{m}$ is given by the solution to

$$
0=\beta\left(T_{m}+\frac{\Gamma}{R}\right)+\frac{(1-c)}{\delta T}\left[\left(T_{m}+\frac{1}{\delta T}\right) \ln \left(T_{m} \delta T+1\right)-T_{m}\right] .
$$

The model dimensionless parameters are defined to be

$$
c=c_{s} / c_{l}, \quad k=k_{s} / k_{l}, \quad \delta T=\Delta T / T_{m}^{*}, \quad \beta=\frac{L_{m}}{c_{l} \Delta T}, \quad \Gamma=\frac{2 \sigma_{s l} T_{m}^{*}}{R_{0} \rho L_{m} \Delta T},
$$

where $\Delta T=T_{H}-T_{m}^{*}$ is the temperature change (with $T_{H}$ the temperature applied at the nanoparticle surface) and $R_{0}$ the initial particle radius.

For water, gold and lead, for a temperature change of $\Delta T=10 \mathrm{~K}$ we find $\beta \approx 8,40,12$ and smaller increases in $\Delta T$ result in larger $\beta$. As a direct consequence of their small volume, the energy required to melt nanoparticles is also small. In fact any increase above the melting temperature on the nanoparticle surface is enough to almost instantaneously melt it. Thus, we focus exclusively on the large Stefan number regime (i.e., $\beta \gg 1$ ). We observe that small $\beta$ suggests a fast melting process as the temperature applied at the nanoparticle surface, $T_{H}$, is much greater than the melting temperature, $T_{m}^{*}$. On the other hand large $\beta$ indicates a slower process as $T_{H}$ is closer to $T_{m}^{*}$. (However, we note that the time-scales are of the order of pico seconds, so slow and fast are relative terms.) This implies a relationship between $\beta$ and the time-scale. Hence we rescale via $t=\beta \tau$ and search for asymptotic solutions of the form $T=T_{0}+T_{1} / \beta+\mathcal{O}\left(1 / \beta^{2}\right)$. In the case of the liquid we have the systems

$$
\begin{aligned}
& \mathcal{O}(1): \quad 0=\frac{1}{r^{2}} \frac{\partial}{\partial r}\left(r^{2} \frac{\partial T_{0}}{\partial r}\right), \quad T_{0}(1, \tau)=1, \quad T_{0}(R, \tau)=T_{m}, \\
& \mathcal{O}(1 / \beta): \quad \frac{\partial T_{0}}{\partial \tau}=\frac{1}{r^{2}} \frac{\partial}{\partial r}\left(r^{2} \frac{\partial T_{1}}{\partial r}\right), \quad T_{1}(1, \tau)=0, \quad T_{1}(R, \tau)=0,
\end{aligned}
$$

with corresponding solutions

$$
\begin{aligned}
& T_{0}=1+\left(T_{m}-1\right) \frac{R}{r}\left(\frac{1-r}{1-R}\right), \\
& T_{1}=\mu_{1}\left\{\left[(3-r) r-\frac{2}{r}\right]-\frac{R}{r}\left(\frac{1-r}{1-R}\right)\left[(3-R) R-\frac{2}{R}\right]\right\} \frac{d R}{d \tau},
\end{aligned}
$$

where

$$
\mu_{1}=\frac{1}{6(1-R)}\left[\frac{\beta \Gamma}{R\left[\beta+\frac{(1-c)}{\delta T} \ln \left(T_{m} \delta T+1\right)\right]}+\frac{\left(T_{m}-1\right)}{(1-R)}\right] .
$$

In the solid

$$
\theta_{0}=T_{m}, \quad \theta_{1}=-\mu_{2}\left(R^{2}-r^{2}\right) \frac{d R}{d \tau}
$$


where

$$
\mu_{2}=\frac{c}{6 k} \frac{\beta \Gamma}{R^{2}\left[\beta+\frac{(1-c)}{\delta T} \ln \left(T_{m} \delta T+1\right)\right]} .
$$

The Stefan condition is

$$
\frac{d R}{d \tau}=\frac{\left(T_{m}-1\right)}{R(1-R)}\left[1+\frac{1}{\beta}\left((1-c) T_{m}-2 \mu_{1} \frac{(1-R)^{2}}{R}-2 k \mu_{2} R\right)\right]^{-1}
$$

and this is coupled to the differentiated version of the Gibbs-Thomson equation

$$
\frac{d T_{m}}{d \tau}=\frac{\Gamma}{R^{2}\left[+\frac{(1-c)}{\beta \delta T} \ln \left(T_{m} \delta T+1\right)\right]} \frac{d R}{d \tau} .
$$

The initial conditions for the above coupled system of equations is $R(0)=1$ and $T_{m}(0)=$ $T_{m}(1)$, where the latter value is fixed by solving (10) with $R=1$. Hence, the problem has been reduced considerably from a system of two heat equations in the solid and liquid defined over a changing domain specified by the Stefan condition and coupled to an equation describing the phase change temperature to solving two first order ODEs.

Figure 2 illustrates the evolution of the melt front, $R(t)$, where the dotted and solid lines are the approximate solution and a numerical solution to the full system, respectively. The three sets of curves denote three associated sets of solutions. Specifically, the curves labelled (i) denote the model using the generalised Gibbs-Thomson relation, curves (ii) take $c_{s}=c_{l}$ in Gibbs-Thomson but not the energy balance (see [23]) and curves (iii) are the standard model where $T_{m}=T_{m}^{*}$ and $c_{s}=c_{l}$. Clearly, the standard model overestimates the melt time considerably. Curves (i) and (ii) show that as the solid radius decreases, the gradient of the curve increases rapidly and tends to infinity. Physically, this implies that in the final stages of melting, the particle will suddenly vanish, and this is the 'abrupt melting' phenomena reported by [6]. Figure 3 presents the temperature profile within the liquid and solid regions as the particle melts. The circles show how the melt temperature decreases with time, the dotted line is the solid phase temperature and the solid line is the liquid temperature. An interesting feature of this graph is the fact that the solid temperature is greater than the phase change temperature. In a standard problem the solid would be be-
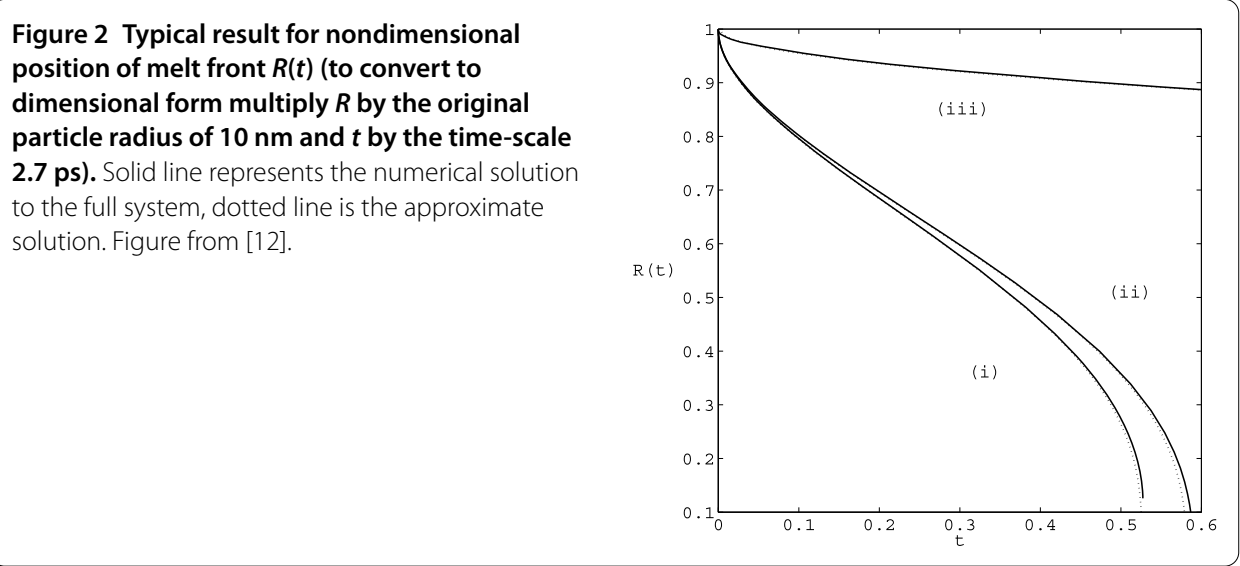
Figure 3 Temperature profiles within a melting

nanoparticle. Figure from [12].

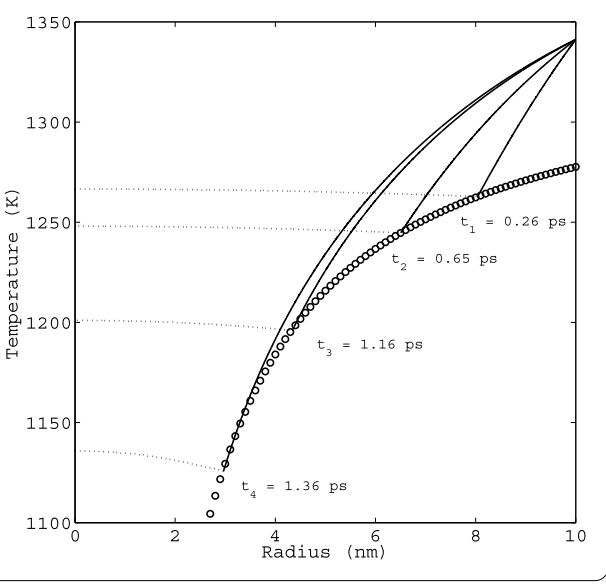

low the phase change temperature and so slow down the process, in this case the decrease in melt temperature means the solid actually acts to speed up the melting.

\section{Thermal conductivity of nanofluids}

The enhanced thermal properties of nanofluids, compared to their base fluids, has led nanofluids to being an attractive solution for heat removal in modern electronic devices $[46,47]$. Consequently, the study of the thermal properties of nanofluids is an intense research area. One of the critical ongoing issues is the lack of a satisfactory theoretical model for the thermal response of a nanofluid [18, 48].

Based on effective medium theory, Maxwell's seminal theoretical description of heat conduction in solid-in-liquid suspensions [49] yields the effective thermal conductivity

$$
\frac{k_{e}}{k_{l}}=\left[\frac{2 k_{l}+k_{p}+2 \phi\left(k_{p}-k_{l}\right)}{2 k_{l}+k_{p}-\phi\left(k_{p}-k_{l}\right)}\right]
$$

where $k_{e}, k_{p}, k_{l}$ denote the effective, particle and liquid thermal conductivity and $\phi$ is the particle volume fraction. The Maxwell model has several well-known drawbacks. Foremost, the model is only applicable to heat flow in the material surrounding an equivalent fluid or around a particle, and not the scenario of interest, namely, an actual nanofluid or particle. Secondly, the theory is based on an infinite region [8] and hence is only applicable to very dilute solutions where the particles are sufficiently far apart such that there is negligible thermal interaction between the particles. Clearly, this will lead to problems if the particle concentration increases. In addition, Maxwell's theory is based on a steadystate analysis, which restricts one from considering the more interesting time dependent thermal response of the nanofluid.

Despite the aforementioned limitations, the Maxwell model works well for low volume fraction fluids with relatively large particles. However, the accuracy of the model deteriorates when the particle size decreases to the nanoscale. Keblinski et al. [50] compared experimental data from various nanofluid treatments and reported that in most cases $k_{e} \approx\left(1+C_{k} \phi\right) k_{l}$ with $C_{k} \approx 5$, which is in contrast to the linearised Maxwell model where $C_{k} \approx 3$. To improve the fit between data and theory, numerous authors have proposed extensions to Maxwell's model including nanolayers (a thin layer of ordered liquid molecules surrounding the particle), particle clustering, nanoconvection and Brownian motion. For 
example, Prasher et al. [48] and Koo and Kleinstreuer [51] modify Maxwell's result to account for Brownian motion. Yu and Choi [52] introduce a nanolayer of width $2 \mathrm{~nm}$ with a thermal conductivity ten times greater than that of the base fluid. The extensive review of Das et al. [8] illustrates that with the inclusion of additional effects and new parameters, there is better agreement between experiment and the variants of Maxwell's theory. In this section we discuss an alternative expression for the thermal conductivity of a nanofluid recently proposed by Myers et al. [13] that agrees well with experimental data.

If we focus on the liquid diffusion time-scale, then a suitable dimensionless model for heat flow through a spherically symmetric liquid-particle system is given by

$$
\begin{array}{ll}
\frac{\partial T}{\partial t}=\frac{\alpha}{r^{2}} \frac{\partial}{\partial r}\left(r^{2} \frac{\partial T}{\partial r}\right), \quad r \in\left[0, r_{p}\right], \\
\frac{\partial \theta}{\partial t}=\frac{1}{r^{2}} \frac{\partial}{\partial r}\left(r^{2} \frac{\partial \theta}{\partial r}\right), \quad r \in\left[r_{p}, 1\right],
\end{array}
$$

where $T$ and $\theta$ are the temperatures in the particle and fluid, respectively, $\alpha=\alpha_{p} / \alpha_{l}$ is the ratio of the particle and liquid thermal diffusivities and $r_{p}$ is the particle radius. We impose a fixed boundary temperature greater than the initial temperature and continuity of temperature and heat flux at the fluid-particle interface. Thus, the relevant conditions are

$$
\begin{array}{ll}
\theta(r, 0)=T(r, 0)=0, \quad \theta(1, t)=1, \quad \theta\left(r_{p}, t\right)=T\left(r_{p}, t\right)=T_{p}(t), \\
\left.\frac{\partial \theta}{\partial r}\right|_{r=r_{p}}=\left.k \frac{\partial T}{\partial r}\right|_{r=r_{p}},\left.\quad \frac{\partial T}{\partial r}\right|_{r=0}=0,
\end{array}
$$

where $k=k_{p} / k_{l}$.

To ascertain the effective thermal conductivity $k_{e}$, we first examine an 'equivalent fluid' with diffusivity $\alpha_{e}$. Hence, we consider the system

$$
\frac{\partial \theta_{e}}{\partial t}=\frac{\alpha_{e}}{r^{2}} \frac{\partial}{\partial r}\left(r^{2} \frac{\partial \theta_{e}}{\partial r}\right), \quad \theta_{e}(1, t)=1,\left.\quad \frac{\partial \theta_{e}}{\partial r}\right|_{r=0}=0,
$$

which is easily solved to give a solution in terms of Bessel's functions. Of particular interest is the temperature at the centre given by

$$
\theta_{e}(0, t)=\theta_{s}(t)=1+\sum_{n=1}^{N} 2(-1)^{n} e^{-n^{2} \pi^{2} \alpha_{e} t} .
$$

The associated particle-fluid system is more complex and requires approximate solution methods. Firstly, we note that to increase the base fluid thermal conductivity, we introduce nanoparticles with a much higher diffusivity relative to the base fluid. For example, copper and $\mathrm{Al}_{2} \mathrm{O}_{3}$ in water or ethylene-glycol solutions, have $\alpha \gg 1$ and $k \gg 1$. In effect, this indicates that heat is transferred more rapidly through the particle than the fluid, and hence $T(r, t) \approx T_{p}(t)$. The fluid thermal problem is reduced to a Cartesian system via the transformation $\theta=u / r$, and the resulting problem is identical to that solved previously via an extension to the Heat Balance Integral Method (HBIM) in $[9,10]$. The HBIM allows for the temperature in the fluid and the particle to be approximated via simple polynomial 
expressions, and thus highlight the influence of the physical parameters in the model. The solution to the HBIM yields

$$
T_{p}=1-e^{-\Lambda\left(t-t_{1}\right)},
$$

where $t_{1}$ is the time when the particle temperature first rises noticeably above the initial temperature, $\Lambda=n \lambda / c_{T}, \lambda=1 /\left(1-r_{p}\right)^{2}$ and $c_{T}=\left(1+r_{p}\right) / 2-1 /(n+1)$. From $[9,10]$ the constant $n=2.233$ is found by minimising the least-squares error when the approximate solution is substituted into the heat equation.

Similarly, we apply the HBIM to the equivalent fluid system to obtain the centre temperature

$$
T_{c}=1-e^{-\Lambda^{\prime}\left(t-t_{1}^{\prime}\right)},
$$

where $\Lambda^{\prime}=n \alpha_{e} / c_{T 0}$ and $c_{T 0}=(n-1) /(2(n+1))$. The accuracy of this formula may be verified by comparison with the exact solution (25).

As the HBIM solution is an acceptable approximation, we find an comparable diffusivity by a matching argument between the HBIM solution for a particle and that of an equivalent fluid. Hence, we adopt a simple approach and equate the decay rates in the expressions for $T_{p}$ and $T_{c}$. This is equivalent to setting $\Lambda=\Lambda^{\prime}$ which yields

$$
\alpha_{e}=\frac{\alpha_{l}}{\left(1-r_{p}\right)^{2}} \frac{n-1}{2(n+1)}\left[\frac{1+r_{p}}{2}-\frac{1}{n+1}\right]^{-1} .
$$

The dimensionless radius $r_{p}$ is scaled with the fluid radius $R$. Typically, these relations are posed in terms of the volume fraction $\phi$ where $r_{p}=\phi^{1 / 3}$, and thus

$$
\alpha_{e}=\frac{\alpha_{l}}{\left(1-\phi^{1 / 3}\right)^{2}} \frac{n-1}{2(n+1)}\left[\frac{1+\phi^{1 / 3}}{2}-\frac{1}{n+1}\right]^{-1} .
$$

A cursory glance of the above expression reveals that the thermal diffusivity of the equivalent fluid depends only on the liquid diffusivity and volume fraction. Critically, the composition of the nanoparticle does not affect $\alpha_{e}$. Via [53], using the relations $\alpha_{e}=k_{e} /(\rho c)_{e}$ and $(\rho c)_{e}=\phi \rho_{p} c_{p}+(1-\phi) \rho_{l} c_{l}$, we may write the effective thermal conductivity as

$$
\frac{k_{e}}{k_{l}}=\frac{\left[(1-\phi)+\phi \frac{\rho_{p} c_{p}}{\rho_{l} c_{l}}\right](n-1)}{\left(1-\phi^{1 / 3}\right)^{2}\left[\left(1+\phi^{1 / 3}\right)(n+1)-2\right]},
$$

which indicates that the equivalent particle conductivity is independent of the particle conductivity. Note this has been verified experimentally, see $[47,52]$. The particle material properties appear through the density and heat, $(\rho c)_{p}$. However, as the ratio $\rho_{p} c_{p} /\left(\rho_{l} c_{l}\right)$ is $\mathcal{O}(1)$ and $\phi$ is small, this is a weak dependence. Consequently, the effective conductivity is predominantly a function of the liquid conductivity $k_{l}$ and volume fraction $\phi$.

To test the validity of our model we compare it to suitable experimental data from the literature. Figure 4 compares the present model (solid line), given by Eq. (30), with that of the Maxwell relation (20) (dashed line) and several experimental data sets for an $\mathrm{Al}_{2} \mathrm{O}_{3}$-water nanofluid [13]. For small volume fractions, the Maxwell relation lies above the present 


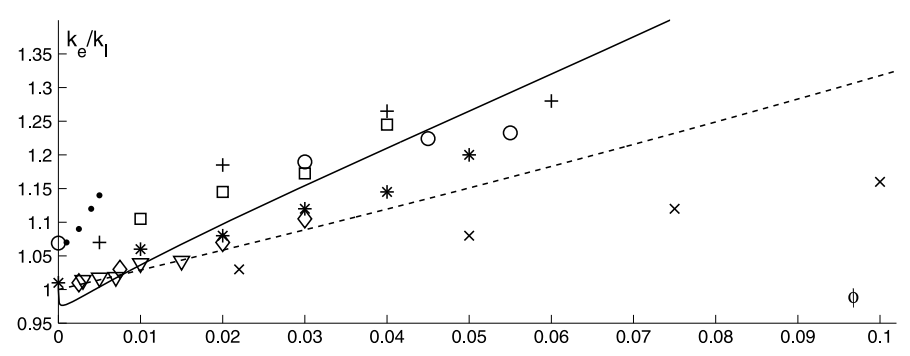

Figure 4 Conductivity ratio $k_{e} / k_{l}$ for $\mathrm{Al}_{2} \mathrm{O}_{3}$-water nanofluid with $\boldsymbol{k}_{l}=\mathbf{0 . 5 8} \mathrm{W} / \mathrm{mK}$. Equation (30) (solid line); Maxwell model equation (20) (dashed line) and experimental data. Figure from [13].

theory and models the data better. However, for $\phi>0.008$, the present model rapidly increases above the Maxwell theory, and more significantly, passes through a larger amount of the data. Thus the present model clearly out performs the basic Maxwell model for the majority of the experimental data for volume fractions greater than $1 \%$.

\section{Conclusion}

This paper provides a brief overview of three pertinent problems from the nano field. In the first problem we considered a model for fluid flow in carbon nanotubes. At present there is no theory for calculating the slip lengths for a liquid moving over a solid surface. We used an equivalent theory with a depletion layer over a solid surface to obtain an expression for the slip length in terms of the depletion layer thickness and its viscosity. Next, we presented a mathematical model that successfully captured the 'abrupt melting' phenomenon of nanoparticles. In particular, the model demonstrated the sharp increase in melting rate as the size of the nanoparticles decreased. Finally, we considered the thermal conductivity of nanofluids, and our analysis led to an expression for solid-in-liquid suspensions, derived in a distinct way to the seminal Maxwell model. The model showed excellent agreement with experimental data for particle volume fractions greater than $1 \%$.

While this work is at the limit of continuum theory, in each case the continuum models demonstrated good agreement with experimental data and provided valuable insight. Moreover, the results of the nanoscale modelling in some cases led to a deeper understanding of the macroscale problems. At present there is great interest in nanotechnology with applications in a wide range of areas. We have presented results for just three specific problems, however the success of the models indicates that there is great scope for applying continuum models at the nanoscale.

\section{Competing interests}

The authors declare that they have no competing interests.

Authors' contributions

All authors contributed equally to the writing of this paper. All authors read and approved the final manuscript.

\section{Author details}

${ }^{1}$ Centre de Recerca Matemàtica, Campus de Bellaterra, Edifici C, Bellaterra, Barcelona, 08193, Spain. ${ }^{2}$ Departament de Matemàtica Aplicada I, Universitat Politècnica de Catalunya, Barcelona, Spain.

\section{Acknowledgements}

The research of TGM was supported by a Marie Curie International Reintegration Grant Industrial applications of moving boundary problems Grant no. FP7-256417 and Ministerio de Ciencia e Innovación Grant MTM2011-23789. VC was also supported through the Marie Curie Grant. FF and MM acknowledge the support of a Centre de Recerca Matemàtica PhD grant. 


\section{References}

1. Abragall P, Nguyen N-T: Nanofluidics. 1st edition. Norwood: Artech House; 2009.

2. Nguyen N-T, Werely ST: Fundamentals and Applications of Microfluidics. 2nd edition. Norwood: Artech House; 2006.

3. Travis KP, Todd BD, Evans DJ: Departure from Navier-Stokes hydrodynamics in confined liquids. Phys Rev E 1997, 55(4):4288-4295

4. Thomas JA, McGaughey AJH, Ottoleo K-A: Pressure-driven water flow through carbon nanotubes: insights from molecular dynamics simulation. Int J Therm Sci 2010, 49(2):281-289.

5. Guisbiers G, Kazan M, Van Overschelde O, Wautelet M, Pereira S: Mechanical and thermal properties of metallic and semiconductive nanostructures. J Phys Chem C 2008, 112:4097-4103.

6. Kofman R, Cheyssac P, Lereah Y, Stella A: Melting of clusters approaching 0D. Eur Phys J D 1999, 9(1-4):441-444.

7. Kuo C-L, Clancy P: Melting and freezing characteristics and structural properties of supported and unsupported gold nanoclusters. J Phys Chem B 2005, 109:13743-13754.

8. Das SK, Choi SU, Yu W, Pradeep T: Nanofluids: Science and Technology. 1st edition. New York: Wiley; 2008.

9. Myers TG: Optimizing the exponent in the heat balance and refined integral methods. Int Commun Heat Mass Transf 2009, 36(2):143-147.

10. Myers TG: Optimal exponent heat balance and refined integral methods applied to Stefan problems. Int J Heat Mass Transf 2010, 53(5-6):1119-1127.

11. Myers TG: Why are slip lengths so large in carbon nanotubes? Microfluid Nanofluid 2011, 10(5):1141-1145 [doi:10.1007/s10404-010-0752-7].

12. Font F, Myers TG: Spherically symmetric nanoparticle melting with a variable phase change temperature. J Nanopart Res 2013, 15(12):2086 [doi:10.1007/s11051-013-2086-3].

13. Myers TG, MacDevette MM, Ribera H: A time-dependent model to determine the thermal conductivity of a nanofluid. J Nanopart Res 2013, 15(7):1775 [doi:10.1007/s11051-013-1775-2].

14. Font F, Myers TG, Mitchell SL: A mathematical model for nanoparticle melting with density change. Microfluid Nanofluid 2014 [doi:10.1007/s10404-014-1423-x].

15. Holt JK, Park HG, Wang Y, Stadermann M, Artyukhin AB, Grigoropoulos CP, Noy A, Bakajin O: Fast mass transport through sub-2-nanometer carbon nanotubes. Science 2006, 312(5776):1034-1037.

16. Majumder M, Chopra N, Andrews R, Hinds BJ: Nanoscale hydrodinamics: enhanced flow in carbon nanotubes. Nature 2005, 438:44.

17. Whitby M, Cagnon L, Thanou M, Quirke N: Enhanced fluid flow through nanoscale carbon pipes. Nano Lett 2008, 8(9):2632-2637.

18. Buongiorno J, Venerus DC, Prabhat N, McKrell T, Townsend J, Christianson R, Tolmachev YV, Keblinski P, Hu L-W, Alvarado JL, Bang IC, Bishnoi SW, Bonetti M, Botz F, Cecere A, Chang Y, Chen G, Chen H, Chung SJ, Chyu MK, Das SK, D Paola R, Ding Y, Dubois F, Dzido G, Eapen J, Escher W, Funfschilling D, Galand Q, Gao J, Gharagozloo PE, Goodson KE, Gutierrez JG, Hong H, Horton M, Hwang KS, lorio CS, Jang SP, Jarzebski AB, Jiang Y, Jin L, Kabelac S, Kamath A Kedzierski MA, Kieng LG, Kim C, Kim J-H, Kim S, Lee SH, Leong KC, Manna I, Michel B, Ni R, Patel HE, Philip J, Poulikakos D, Reynaud C, Savino R, Singh PK, Song P, Sundararajan T, Timofeeva E, Tritcak T, Turanov AN, Van Vaerenbergh S, Wen D, Witharana S, Yang C, Yeh W-H, Zhao X-Z, Zhou S-Q: A benchmark study on the thermal conductivity of nanofluids. J Appl Phys 2009, 106(9):094312.

19. MacDevette MM, Myers TG, Wetton B: Boundary layer analysis and heat transfer of a nanofluid. Microfluid Nanofluid 2014, 17(2):401-412 [doi:10.1007/s10404-013-1319-1].

20. Thamwattana N, Hill JM, Baowan D, Cox BJ: A review of mathematical and mechanical modelling in nanotechnology. Math Mech Solids 2010, 15:708-717.

21. Wu T, Liaw H-C, Chen Y-Z: Thermal effect of surface tension on the inward solidification of spheres. Int J Heat Mass Transf 2002, 45(10):2055-2065.

22. Wu B, McCue SW, Tillman P, Hill JM: Single phase limit for melting nanoparticles. App/ Math Model 2009, 33(5):2349-2367.

23. McCue SW, Wu B, Hill JM: Micro/nanoparticle melting with spherical symmetry and surface tension. IMA J App/ Math 2009, 74:439-457.

24. Meyyappan M: Carbon Nanotubes: Science and Applications. 1st edition. Boca Raton: CRC Press; 2004.

25. Pantarotto D, Singh R, McCarthy D, Erhardt M, Briand J-P, Prato M, Kostarelos K, Bianco A: Functionalized carbon nanotubes for plasmid DNA gene delivery. Angew Chem, Int Ed Engl 2004, 43(39):5242-5246 [doi:10.1002/anie.200460437].

26. Wang J: Carbon-nanotube based electrochemical biosensors: a review. Electroanalysis 2005, 17(1):7-14

27. Zanello LP, Zhao B, Hu H, Haddon RC: Bone cell proliferation on carbon nanotubes. Nano Lett 2006, 6(3):562-567.

28. Suzuki K, Yamaguchi M, Kumagai M, Yanagida S: Application of carbon nanotubes to counter electrodes of dye-sensitized solar cells. Chem Lett 2003, 32(1):28-29 [doi:10.1246/cl.2003.28].

29. Heinze S, Tersoff J, Martel R, Derycke V, Appenzeller J, Avouris P: Carbon nanotubes as Schottky barrier transistors. Phys Rev Lett 2002, 89:106801 [doi:10.1103/PhysRevLett.89.106801].

30. White FM: Viscous Fluid Flow. 1st edition. New York: McGraw-Hill; 1991

31. Thomas JA, McGaughey AJH: Reassessing fast water transport through carbon nanotubes. Nano Lett 2008, 8(9):2788-2793.

32. Verweij H, Schillo MC, Li J: Fast mass transport through carbon nanotube membranes. Small 2007, 3(12):1996-2004

33. Cottin-Bizonne C, Cross B, Steinberger A, Charlaix E: Boundary slip on smooth hydrophobic surfaces: intrinsic effects and possible artifacts. Phys Rev Lett 2005, 94:056102.

34. Eijkel JT, van den Berg A: Nanofluidics: what is it and what can we expect from it? Microfluid Nanofluid 2005, 1(3):249-267.

35. Neto C, Evans DR, Bonaccurso E, Butt H-J, Craig VSJ: Boundary slip in Newtonian liquids: a review of experimental studies. Rep Prog Phys 2005, 68(12):2859 [http://stacks.iop.org/0034-4885/68/i=12/a=R05].

36. Poynor A, Hong L, Robinson IK, Granick S, Zhang Z, Fenter PA: How water meets a hydrophobic surface. Phys Rev Lett 2006, 97:266101 [doi:10.1103/PhysRevLett.97.266101]. 
37. Joseph S, Aluru NR: Why are carbon nanotubes fast transporters of water? Nano Lett 2008, 8(2):452-458.

38. Barrat J-L, Bocquet $L$ : Influence of wetting properties on hydrodynamic boundary conditions at a fluid/solid interface. Faraday Discuss 1999, 112:119-128.

39. Matthews MT, Hill JM: Nanofluidics and the Navier boundary condition. Int J Nanotechno/ 2008, 5(2/3):218-242.

40. Choi C-H, Westin KJA, Breuer KS: Apparent slip flows in hydrophilic and hydrophobic microchannels. Phys Fluids 2003, 15(10):2897-2902.

41. Shim J-H, Lee B-J, Cho YW: Thermal stability of unsupported gold nanoparticle: a molecular dynamics study. Surf Sci 2002, 512:262-268.

42. Buffat $P$, Borel JP: Size effect on the melting temperature of gold particles. Phys Rev A 1976, 13(6):2287-2297.

43. Bergese P, Colombo I, Gervasoni D, Depero LE: Melting of nanostructured drugs embedded into a polymeric matrix. J Phys Chem B 2004, 108:15488-15493.

44. Liu X, Yangb P, Jiang Q: Size effect on melting temperature of nanostructured drugs. Mater Chem Phys 2007, 103:1-4.

45. Rana S, Bajaj A, Mout R, Rotello VM: Monolayer coated gold nanoparticles for delivery applications. Adv Drug Deliv Rev 2012, 64:200-216

46. Nguyen CT, Roy G, Gauthier C, Galanis N: Heat transfer enhancement using $\mathrm{Al}_{2} \mathrm{O}_{3}$-water nanofluid for an electronic liquid cooling system. App/ Therm Eng 2007, 27(8-9):1501-1506 [doi:10.1016/j.applthermaleng.2006.09.028].

47. Yu W, France DM, Routbort JL, Choi SUS: Review and comparison of nanofluid thermal conductivity and heat transfer enhancements. Heat Transf Eng 2008, 29(5):432-460.

48. Prasher R, Bhattacharya P, Phelan PE: Thermal conductivity of nanoscale colloidal solutions (nanofluids). Phys Rev Lett 2005, 94:025901.

49. Maxwell JC: A Treatise on Electricity and Magnetism. Volume 1. 3rd edition. Oxford: Clarendon Press; 1881

50. Keblinski P, Eastman JA, Cahill DG: Nanofluids for thermal transport. Mater Today 2005, 8(6):36-44

51. Koo J, Kleinstreuer C: A new thermal conductivity model for nanofluids. J Nanopart Res 2004, 6(6):577-588 [doi:10.1007/s11051-004-3170-5].

52. Yu W, Choi SUS: The role of interfacial layers in the enhanced thermal conductivity of nanofluids: a renovated Maxwell model. J Nanopart Res 2003, 5(1-2):167-171 [doi:10.1023/A:1024438603801].

53. Zhou S-Q, Ni R: Measurement of the specific heat capacity of water-based $\mathrm{Al}_{2} \mathrm{O}_{3}$ nanofluid. Appl Phys Lett 2008 92(9):093123.

$10.1186 / 2190-5983-4-11$

Cite this article as: Myers et al.: Continuum mathematics at the nanoscale. Journal of Mathematics in Industry 2014, $4: 11$

\section{Submit your manuscript to a SpringerOpen ${ }^{\ominus}$ journal and benefit from:}

- Convenient online submission

- Rigorous peer review

- Immediate publication on acceptance

- Open access: articles freely available online

- High visibility within the field

- Retaining the copyright to your article 